\title{
Neural representation in F5: cross-decoding from observation to execution
}

\author{
Murat Kirtay ${ }^{1}$, Vassilis Papadourakis ${ }^{2,3}$, Vassilis Raos ${ }^{2,3}$, Erhan Oztop ${ }^{1^{*}}$ \\ From 24th Annual Computational Neuroscience Meeting: CNS*2015 \\ Prague, Czech Republic. 18-23 July 2015
}

Mirror neurons fire during both action execution and observation of a similar action performed by another individual [1]. However, this definition does not account for the existence of representational equivalence between execution and observation. To investigate this issue we recorded 68 neurons from area F5 of a macaque monkey trained either to execute reaching-to-grasp actions towards objects or to observe the experimenter performing the same actions [2], and adopted a decoding framework to find whether neurons effective in decoding the object/grip type in (1) execution and (2) observation conditions do exist, and most critically, to (3) assess whether transfer between execution and observation decoders (i.e. cross-decoding) can be employed. By 'transfer' we mean the application of the decoder parameters estimated using the neural discharge in observation to the neural firing recorded in execution, and vice versa. The success rate of such a decoder indicates the equivalence of representations in the two conditions.

Our analysis indicates that, at the level of single neurons, object/grip-specific decoders can be constructed, i.e. the type of the object/grip employed in either execution or observation can be decoded (success rate: $80 \%-100 \%$, chance level: $25 \%$ ). However, only in $10 \%$ of the cases (corresponding to the congruent type mirror neurons [1]) the decoder based on the execution discharge was effective when transferred to the observation discharge. The same was true for the reverse transfer. To extend this analysis at the population level we examined all pair performance of a 10-neuron set, consisted of 4 neurons having the best decoding performance and 6 neurons randomly selected. Out of the 45 possible pairs, 7 displayed high success rates ( $80 \%$ on average) in cross-decoding. Remarkably, high performing pairs were constituted only when one of the

* Correspondence: erhan.oztop@ozyegin.edu.tr

${ }^{1}$ Computer Science, Ozyegin University, Istanbul, Turkey

Full list of author information is available at the end of the article neurons displaying reliable decoding performance was paired with a randomly selected -poor solo decoder- neuron, which acted as a "helper". These results strongly point to a population based representation where good and poor decoders may cooperate to form a robust recognition system.

\section{Methods}

Neuronal discharges during each condition were trimmed and represented as 14-bin histogram vectors. In the two neuron analysis, each neuron was reduced to a 7-bin histogram, and their concatenation results in a 14-tuple vector, to ensure similar decoder complexity (constant number of adjustable parameters). Thus, for each condition, ten 14-tuple neural firings (one per trial) made up the rows of the input matrix $\mathbf{X}$, and the corresponding object ids (1-4) made up the output vector $\mathbf{Y}$. We assumed a linear relation between input and output as $\mathbf{X W}=$ Yand solved for the weights (the decoder parameters) using the pseudo-inverse solution $\mathbf{W}=\mathbf{X}^{\dagger} \mathbf{Y}$. Then, given a 14-tuple vector representation, $\mathbf{z}$, of a discharge, the predicted object id is given by $y_{\text {pred }}=\underset{i=0 . .5}{\arg \min }\left\{[0,1,2,3,4,5]-\mathbf{z}^{T} \mathbf{W}\right\}$, where 0 and 5 indicates a definite wrong prediction. For executiononly and observation-only experiments, leave-one-out cross validation was applied to obtain the success rates in decoding. For cross-decoding analysis, the weight vector $\mathbf{W}$ obtained in one condition was used to predict the object type in the other condition by using the data from that condition.

\section{Acknowledgements}

This work was supported by the grant "OBSERVENEMO" within the framework of the bilateral S\&T Cooperation Program between the Republic of Turkey and the Hellenic Republic. Grant no 1135391 funded by TUBITAK and grant ГГET 14TUR OBSERVENEMO co- Financed by the European Union and the Greek State, MCERA/GSRT. 


\section{Authors' details}

${ }^{1}$ Computer Science, Ozyegin University, Istanbul, Turkey. ${ }^{2}$ Foundation for Research \& Technology- Hellas (FORTH), Heraklion, Greece. ${ }^{3}$ University of Crete Medical School, Heraklion, Greece.

Published: 18 December 2015

\section{References}

1. Fadiga L, Fogassi L, Rizzolatti G: Action recognition in the premotor cortex. Brain 1996, 119:593-609.

2. Papadourakis V, Raos V: Cue-dependent action-observation elicited responses in the ventral premotor cortex (area F5) of the macaque monkey. Soc Neurosci Abstr 2013, Program No. 263.08.

doi:10.1186/1471-2202-16-S1-P190

Cite this article as: Kirtay et al:: Neural representation in F5: crossdecoding from observation to execution. BMC Neuroscience 201516 (Suppl 1):P190.

Submit your next manuscript to BioMed Central and take full advantage of:

- Convenient online submission

- Thorough peer review

- No space constraints or color figure charges

- Immediate publication on acceptance

- Inclusion in PubMed, CAS, Scopus and Google Scholar

- Research which is freely available for redistribution

Submit your manuscript at www.biomedcentral.com/submit
C Biomed Central 